\section{Prostatakrebs: bessere Prognose nach postoperativer Radiatio}

\begin{abstract}
Prostatakarzinompatienten haben selbst zehn Jahre nach einer radikalen Prostatektomie und anschließender Bestrahlung eine bessere Prognose als Patienten ohne postoperative Radiatio. Das gilt vor allem für jüngere Männer. Anders als nach fünf Jahren wirkt sich die Bestrahlung allerdings nicht auf das klinische progressionsfreie Überleben und das Gesamtüberleben aus, sondern vor allem auf das biochemische progressionsfreie Überleben, definiert anhand des PSA-Wertes.
\end{abstract}

$\mathrm{V}$ on der europäischen EORTC-Studie 22911 wurden nun die Zehn-JahresDaten vorgestellt. Für die Studie wurden Daten von mehr als 1.000 Prostatakarzinompatienten ausgewertet, die postoperativ innerhalb von vier Monaten oder nicht bestrahlt worden waren. Bestrahlt wurde mit einer Dosis von 50 Gy in 25 Fraktionen über einen Zeitraum von fünf Wochen. Zur „Boosterung“ wurden 10 Gy in fünf Fraktionen über eine Woche verabreicht. Die Follow-up-Dauer lag im Median bei knapp elf Jahren, mit einer Spanne von zwei Monaten bis zu 16,6 Jahren.
Im Vergleich zu den Männern ohne Bestrahlung verbesserte die Radiatio das biochemische progressionsfreie Überleben signifikant. 39,4\% der Patienten erfuhren eine biochemische oder klinische Progression oder starben, im Vergleich zu 61,8\% der Patienten in der Überwachungsgruppe. Das entspricht einer Hazard Ratio von 0,49. Damit wurden die Ergebnisse der FünfJahres-Auswertung bestätigt.

Als biochemische Progression wurde gewertet, wenn der PSA-Wert über 0,2 $\mathrm{ng} / \mathrm{ml}$ stieg. Der mediane PSA-Wert betrug vor der Prostatektomie zwischen 12,3 $\mathrm{ng} / \mathrm{ml}$ und $12,5 \mathrm{ng} / \mathrm{ml}$, nach der Operati- on und vor der Bestrahlung 0,2 ng/ml. Allerdings geht aus der Auswertung der EORTC-Studie auch hervor, dass unerwünschte Wirkungen im späteren Verlauf der Nachbeobachtungszeit in der Gruppe mit Bestrahlung häufiger waren als in der Vergleichsgruppe, und zwar mit einer kumulativen Inzidenz nach zehn Jahren von $70,8 \%$ versus $59,7 \%(p=0,001)$. Die Bestrahlung hatte keinen Einfluss auf die Gesamtüberlebensrate, auf das klinische progressionsfreie Überleben und auf die Entwicklung von Fernmetastasen.

Fazit: Möglicherweise profitieren Männer, die jünger als 70 Jahre sind, mehr von der Bestrahlung, sodass auch das klinische progressionsfreie Überleben verlängert wird, vermuten die Autoren aufgrund der Studiendaten. Bei älteren Männern könnte die Behandlung eher schädlich sein.

Peter Leiner

Bolla $\mathrm{M}$ et al. Postoperative radiotherapy after radical prostatectomy for high-risk Prostate cancer: long-term results of a randomized controlled trial (EORTC trial 22911). Lancet 2012; 380: 2018-27

\section{Protonen kaum weniger toxisch als IMRT}

Weil sich mit der Protonentherapie Tumoren gezielt bestrahlen lassen, ist das Interesse daran auch für das Prostatakarzinom groß. In den USA haben Radiologen jetzt geprüft, ob sie weniger Nebenwirkungen hat als die intensitätsmodulierte Radiotherapie (IMRT).

W ie in Deutschland wird die Protonentherapie in den USA im Vergleich zur etablierten intensitätsmodulierten Radiotherapie (IMRT) bisher nur an wenigen spezialisierten Zentren angeboten. Aus früheren US-amerikanischen Studien gibt es Hinweise, dass es mit der IMRT weniger gastrointestinale Toxizitäten gibt als mit der Protonentherapie. Allerdings sind die Daten nicht für die ganzen USA repräsentativ. Um den Langzeiteffekt der beiden Therapieformen bei Männern mit einem noch nicht metastasierten Prostatakarzinom zu ermitteln, werteten jetzt USRadiologen Daten aller Medicare-Patienten älter als 65 Jahre im Zeitraum zwischen 2008 und 2009 aus.
Von 27.647 Männern mit Prostatakarzinom erhielten 553 eine Protonentherapie (2\%) und 27.100 eine IMRT (98\%). Auffallend waren toxische Wirkungen vor allem im Urogenitalbereich. Ihr Anteil lag zwar sechs Monate nach Therapie bei Männern mit Protonentherapie signifikant niedriger als bei Männern mit IMRT (5,9\% vs. $9,5 \%, p=0,03)$, doch nach einem Jahr war der Unterschied nicht mehr signifikant $(p=0,66)$. Die Unterschiede bei gastrointestinalen Toxizitäten waren über den gesamten Studienzeitraum nicht signifikant.

Die Autoren weisen aber darauf hin, dass ihnen Informationen über die Strahlendosis für die Auswertung fehlten. Möglicherweise sei die Dosierung bei der
IMRT höher gewesen, was die höhere Toxizität sechs Monate nach Studienbeginn erklären könnte. Eventuell könnte die Ursache aber auch darin liegen, dass die Bestrahlung per IMRT eher regional als bei der Protonentherapie ist, die gezielt die Prostata im Fokus hat. Solange es keine prospektiven Vergleichsstudien gebe, sei es nicht gerechtfertigt, die Protonentherapie flächendeckend anzubieten, so die Autoren.

Fazit: Die Toxizität nach Protonentherapie bei Männern mit nicht metastasiertem Prostatakarzinom ist zumindest in dieser US-amerikanischen Studie nur unwesentlich niedriger als nach IMRT. Noch gibt es aber zu wenig Daten aus randomisierten Vergleichsstudien, um einen klaren klinischen Vorteil der Protonentherapie zu belegen.

Peter Leiner

Yu JB et al. Proton Versus IntensityModulated Radiotherapy for Prostate Cancer: Patterns of Care and Early Toxicity J Natl Cancer Inst 2013; 105: 25-32 